\title{
Comparison between stellarator and tokamak divertor transport
}

\author{
Y. Feng, M. Kobayashi*, T. Lunt, D. Reiter** \\ Max-Planck-Institute fuer Plasmaphysik, Germany \\ *National Institute for Fusion Science, Toki, Japan \\ ** Institute for Energy Research-Plasma Physics, Forschungszentrum Jülich, Germany
}

\begin{abstract}
The paper compares the essential divertor transport features of the poloidal divertor, which is well-developed for tokamaks, and the non-axisymmetric divertors currently investigated on helical devices. It aims at surveying the fundamental similarities and differences in divertor concept and geometry, and their consequences for how the divertor functions. In particular, the importance of various transport terms governing axisymmetric and helical scrape-off-layers (SOLs) is examined, with special attention being paid to energy, momentum and impurity transport. Tokamak and stellarator SOLs are compared by identifying key geometric parameters through which the governing physics can be illustrated by simple models and estimates. More quantitative assessments rely nevertheless on the modeling using EMC3-EIRENE code. Most of the theoretical results are discussed in conjunction with experimental observations.
\end{abstract}

\section{Introduction}

Although the divertor idea within the stellarator concept was suggested by Spitzer already in the 50's of the last century [1,2], intensive exploration of viable divertors for stellarators was started only recently. In contrast, divertor programs in tokamaks began much earlier. After extensive joint research in the tokamak community over several decades, the poloidal divertor as a successful concept has been accepted by most of the existing tokamaks (see e.g. $[3,4]$ and the references therein) and by ITER as well $[5,6,7]$. Divertors for both tokamaks and stellarators follow the same principle, i.e. separating the plasma-surface interaction region topologically from the confinement core by applying appropriate separatrix-bounded magnetic configurations. They aim at similar goals and share the same technology. Divertor transport and physics are subject to the same atomic and plasma-surface interaction processes and the same fundamental plasma transport processes. In this regard, divertor research recently started on stellarators benefits from the experience and knowledge in both technology and physics gathered in the tokamak community. On the other hand, however, significant differences in divertor geometry and magnetic configuration exist between helical and axisymmetric devices, which influence the plasma, neutral and impurity transport in the SOL and consequently the functionality of a divertor. From this point of view, stellarators open a new window for exploring the optimal divertor solutions for magnetic confinement fusion devices on a broader basis.

Unlike the poloidal divertor in tokamaks, where a separatrix is formed by introducing additional polodial fields, divertor configurations currently explored in helical devices are based on specific edge magnetic structures intrinsically available in each device. Typical examples are the island divertor (ID) for the advanced low-shear stellarators W7-AS and W7-X [8-10], and the local island (LID) [11, 12] and the helical divertor (HD) [13, 14] for the 
high-shear, largest heliotron-type device LHD. An overview of the divertor activities in stellarators is given in [15].

The poloidal divertor preserves the tokamak toroidal symmetry. Smooth flux surfaces can be constructed and ordered in the SOL to provide a natural coordinate-basis for 2D SOL transport modeling [16-19]. In contrast, the divertor configurations in stellarators exhibit helical structures locally interacting with 3D-shaped divertor plates (see e.g. the W7-AS island divertor shown in figure 1). Helical SOLs in stellarators are therefore fully $3 \mathrm{D}$ and the field lines usually exhibit certain types of stochastic behaviour depending on the field spectrum and the shear at the edge in individual devices. Thus, SOL transport models for helical devices need to meet the increased dimensionality and face the difficulty of dealing with a stochastic field where flux surfaces do not exist. Different concepts and strategies have been explored [20-22]. One example is the EMC3-EIRENE[22, 23] code. It is employed in this paper for physics interpretation of $3 \mathrm{D}$ divertor transport and therefore needs a short introduction. EMC3 is a 3D fluid code for both the background plasma and impurities, and solves the fluid equations by applying a Monte-Carlo method on a locally field-aligned vector basis [24]. EIRENE is a 3D kinetic Monte Carlo Code for neutral particles, radiation transfer and kinetic trace ion impurity transport [23]. EMC3-EIRENE was initially developed and applied for W7-AS $[22,25-29]$ and has recently found applications to 3D edge transport problems encountered not only in stellarators [30,31] but also in tokamaks [32-37].

The paper compares the essential divertor transport features of axisymmetric and helical devices. It aims at surveying the fundamental similarities and differences in divertor concept and geometry, and their consequences on the basic function elements of a divertor. Stellarator-specific effects are emphasized while most of the tokamak SOL phenomena are considered to be known with the details being referred to in the literature. Throughout the paper the discussion is guided by simple models and estimates, and the numerical modelling serves to verify their self-consistency. Certain devices and divertor configurations are chosen as examples for the discussion, but the principal conclusions are not restricted to these devices.

\section{Large variety of divertor configurations}

In a divertor configuration, particle and energy leaving the confinement region are guided by open field lines to targets which are remote from the confinement core. In the poloidal divertor of tokamaks the nested flux surfaces are opened at the edge by creating additional poloidal fields of comparable strength to that generated by the plasma current. In the island divertor, magnetic flux surfaces are torn apart by magnetic islands resulting from a small radial field component resonant to a rational surface. The magnetic islands provide a natural separatrix configuration without need of any additional fields. The geometric principles of the tokamak poloidal-field divertor and of the divertors in helical devices are sketched in figure 2. Low-shear stellarators like the W7-family allow only for the existence of a single island chain at the edge. The rotational transform in the edge region of the LHD HD configurations covers many resonances which overlap and form a stochastic layer of $\sim 10 \mathrm{~cm}$ thickness. Unlike the single island chains in W7-AS and W7-X, the stochastic SOL in LHD exhibits a complex field structure characterized by the coexistence of remnant magnetic islands, stochastic fields and edge surface layers [14]. In the outermost region close to the wall, the increased poloidal field components of the two helical coils create 4 divertor legs which are cut by graphite targets positioned just in front of the wall, forming a divertor configuration similar to the double-null configuration in tokamaks. Nevertheless, the divertor 
target probes measure rather low downstream densities, typically $<10^{19} \mathrm{~m}^{-3}$, even in high-density operation in LHD [38]. Regarding neutral screening in the present open divertor configuration, the pre-X-point stochastic layer is much "thicker" than the divertor legs and builds the major part of the HD SOL. In this paper, our attention will be paid only to the stochastic layer.

Target plates are usually shaped to meet the basic geometry and symmetry of the magnetic field configuration provided in each device. For example, the poloidal-field divertor target in tokamaks is axisymmetric, while the divertor plates in stellarators have a helical form.

Particles and energy entering the SOL are transported toward the targets along the fastest available transport channel. Transport along field lines is considered to be classical whereas cross-field transport processes are assumed to be anomalous. In tokamak SOLs, parallel transport processes clearly dominate and are characterized by the field-line connection length and ion acoustic speed. For describing the divertor transport in stellarators, the situation is more subtle and additional geometric parameters are needed for the following reasons. The contribution of parallel motion to the divertor transport results from a finite field-line pitch $\Theta$. In tokamaks this pitch arises from the external poloidal fields (poloidal divertor), i.e. $\Theta=B_{p} / B$, and takes a typical value of 0.1 . For the island divertor, the divertor-relevant field-line pitch (perpendicular displacement of a field line to targets per field-line length) arises from a much smaller radial component $\mathrm{B}_{\mathrm{r}}(\mathrm{m}, \mathrm{n})$ resonant to the $\mathrm{n} / \mathrm{m}$ rational surface forming the islands, i.e. $\Theta=B_{\mathrm{r}} / \mathrm{B}$, typically in the order of $10^{-3}$. The connection length (X-point-to-X-point) in tokamaks can be estimated by $L_{c}=2 \pi R / N \imath$, where $N$ is the null-number and $\imath$ the rotational transform. A representative connection length for an island divertor can be expressed by $L_{c}=2 \pi R / m \cdot r_{i} \cdot \imath^{\prime}$ [27], where $\mathrm{m}$ is the poloidal number of the islands, $r_{i}$ the radial island width and $\imath^{\prime}$ the shear around the $\mathrm{n} / \mathrm{m}$ resonance. Thus, the connection length in an island divertor is determined by the shear and is usually much larger than in tokamaks of similar size. The open field lines in the stochastic layer exhibit a rather complex evolution structure, yielding a connection length contour ranging from several $\mathrm{m}$ to several $\mathrm{km}$, as shown in figure 3 [41]. The outermost region is dominated by field lines of short connection lengths. There exist, however, multiple edge surfaces filled by long field lines of several $100 \mathrm{~m}$ connection length, forming the main plasma parallel transport channels across the stochastic layer. Thus, the characteristic perpendicular-to-parallel transport scale-length ratio in LHD, i.e. $10 \mathrm{~cm}$ SOL thickness divided by several $100 \mathrm{~m}$ connection length, is even smaller than those in the IDs of W7-AS and W7-X. For these reasons, the cross-field transport is relatively more important in helical SOLs. In addition, the divertor legs in tokamaks are isolated from each other in terms of cross-field transport (ignoring possible contributions from neutral gas). As a consequence, the plasma pressure (static+dynamic) is constant along the field lines in non-detached divertors. In contrast, in stellarators the SOL often contains regions which are situated close to each other but where the plasma flows in opposite directions. If these regions come close enough to one another, cross-field transport can efficiently transfer momentum from one such channel to another.

\section{Basic transport features of tokamaks and stellarator}

\subsection{Experimental observations}

In view of the large differences in the most important geometric parameters, different divertor transport behaviour must be expected in stellarators and tokamaks. This is indeed 
observed experimentally. As an example for tokamaks, figure 4 shows results from ASDEX published by Shimomura et al in 1983 [39]. From the $\mathrm{n}_{\text {ed }}$ (downstream density)-behaviour, different SOL transport regimes may be identified (see [39] for details). When the line-averaged density in the plasma core is increased, $\mathrm{n}_{\mathrm{ed}}$ rises slowly at the beginning and then grows more rapidly. After reaching a maximum, ned drops at even higher upstream density. The three operational regimes of different $\mathrm{n}_{\mathrm{ed}}$-scalings are referred to in the literature as linear (or sheath-limited), high-recycling (or conduction-limited) and (fully recognized only a decade after this experimental finding) as "detachment regime", respectively.

Figure 5 shows the experimental results from the first island divertor explored on W7-AS around 2000 [9], in comparison with EMC3-EIRENE modelling [25]. Without going into details of the experimental results, one sees that a clear transition from the linear to the high-recycling regime accompanied by a sharp change in $\mathrm{n}_{\text {ed }}$-slope as observed in tokamaks (see e.g. figure 4) does not occur in W7-AS, nor is it found by EMC3-EIRENE modelling. Instead, $n_{\text {ed }}$ follows $n_{\text {es }}$ almost linearly up to a certain point of "roll-over". Similar to the W7-AS results, a high-recycling regime is not found in LHD, either experimentally or numerically. Prior to detachment, both experimental and numerical results usually show a roughly linear coupling between $\mathrm{n}_{\mathrm{ed}}$ and $\mathrm{n}_{\mathrm{es}}$, with $\mathrm{n}_{\mathrm{ed}}$ far below $\mathrm{n}_{\mathrm{es}}$ even at the roll-over point [40, 41]. Figure 6 shows the typical behaviour of the ion saturation currents from the target Langmuir probes over a density scan in the helical divertor of LHD. $\mathrm{I}_{\text {sat }}$ increases linearly prior to roll-over and drops at high density. In an attempt to model this behaviour, EMC3-EIRENE simulations have been carried out with different choices for the perpendicular transport coefficients. A noticeable change in the $\mathrm{I}_{\mathrm{sat}}$-slope prior to roll-over cannot be identified, independently of the details of the cross-field transport coefficients that are assumed.

\subsection{Governing transport terms in stellarators and tokamaks}

The significant differences in divertor transport observed in stellarators and tokamaks motivate a rough estimate of the basic physics mechanism involved in the various transport channels. To this end, we need first to simplify the divertor geometry and reduce the dimensionality of the problem, as shown in figure 7. Similar sketches for the tokamak poloidal divertor can be found readily in the tokamak literature, e.g. [4]. Only one point needs to be emphasized here: the background plasma ions flowing along the two divertor legs (single-null) have opposite toroidal velocities. Replacing the 'U'-form SOL of the single-null poloidal divertor by a single island chain, one obtains a schematic SOL for the island divertor in W7-AS and W7-X, as shown by the right sketch in figure 7. For LHD, we use figure 3 as reference.

Following the basic idea behind the two-point models for tokamaks [42-44], we develop a 1D model to schematically describe both the stellarator and tokamak SOLs. We use the simplified SOL geometries shown in figures 7 and take the "shortest" perpendicular distance to the target, "x", as our only coordinate. For the LHD stochastic layer shown in figure 3, this coordinate is $r_{\text {eff }}$ instead. Parallel motion enters the problem through the finite field-line pitch $\Theta=d x / d l_{\| /}$with $l_{l /}$ being the arc length along the field. Of course, $\Theta$ is a function of the two or three coordinates in a realistic SOL, but for simplicity we assume a spatially constant $\Theta$. The $1 \mathrm{D}$ transport model then becomes 


$$
\begin{aligned}
& \Theta \frac{d}{d x}\left(-\kappa_{e, i} T_{e, i}^{5 / 2} \Theta \frac{d T_{e, i}}{d x}\right)+\frac{d}{d x}\left(-\chi_{e, i} n \frac{d T_{e, i}}{d x}\right)=0 \\
& \Theta \frac{d}{d x}\left(n V_{\mathrm{ill}}^{2}+\frac{p}{m_{i}}\right)=-\frac{d}{d x}\left(-D \frac{d}{d x} n V_{\mathrm{ill}}\right)-D \frac{n V_{\mathrm{ill}}}{\Delta y^{2}} \\
& n_{d} c_{s d} \gamma T_{d}=q_{\mathrm{II}}
\end{aligned}
$$

Eq (1) includes the parallel and perpendicular conductive heat transport fluxes for electrons and ions with $n=n_{e}=n_{i}$ and $\chi_{e, i}$ being the perpendicular thermal diffusivities. Eq (2) is the parallel momentum balance where $V_{i l l}$ is the parallel flow velocity and $p$ is the total thermal pressure of ions and electrons. The last two terms in eq (2) represent cross-field momentum transport, which is relevant only for helical SOLs, as will be explained later in more detail. Equation (3) is the Bohm-condition at targets where the subscript ' $d$ ' means downstream. We first focus on eq (1) and compare the parallel conductive heat flux with the perpendicular one for electrons and ions. For a spatially constant field-line pitch $\Theta$, the ratio of the parallel to the perpendicular heat flux is given by

$$
\text { II-heat flux } \perp \text {-heat flux }=\kappa_{e, i} T_{e, i}^{5 / 2} \Theta^{2} / \chi_{e, i} n \text {. }
$$

Stellarators differ from tokamaks through the value of $\Theta$. For $\chi_{e}=\chi_{i}=2 \mathrm{~m}^{2} / \mathrm{s}$, figure 8 shows the $I I / \perp$-ratios in the typical SOL parameter range relevant for both stellarators and tokamaks. The four curves represent the $I V \perp=1$ conditions for ions and electrons in a typical tokamak $(\Theta=0.1)$ and a stellarator $(\Theta=0.001)$, respectively. The two tokamak curves lie in the region close to the lower-right corner of the diagram, implying that, in most of the SOL parameter domain, ||$/ \perp>>1$ holds in tokamaks, especially for electrons. Because of the small $\Theta$, the perpendicular and parallel transport are much more comparable in a stellarator SOL, even for electrons. In fact, as shown in figure 8, the II-to- $\perp$ transport ratio in stellarators for both ions and electrons can be tuned from $>1$ to $<1$ in experiments either internally by varying the SOL plasma parameters or by externally adjusting $\Theta$. This fine-tuning of the SOL transport has turned out to have a strong impact on impurity transport and the stability of detached plasmas. We will return to this point later.

The two divertor legs of the single-null poloidal divertor in tokamaks form two parallel channels in which plasma ions move in opposite toroidal directions onto the respective targets. The two channels are isolated from each other in terms of cross-field transport. In other words, the characteristic cross-field transport length scale is much shorter than the geometrical channel separation. One could image what would happen if instead the two channels would approach each other and even partially overlap. Intense momentum exchange would be expected to take place between the two channels leading to mutual momentum loss of the counter-streaming ions in the neighbouring channels. The resulting friction force must be balanced by the pressure-gradient force. As a consequence, the up-/downstream pressure ratio increases. This is what is believed to happen in helical SOLs. Figure 9 shows a typical flow pattern in W7-AS calculated by the EMC3-EIRENE code. Counter-flows residing on neighbouring island fans effectively touch each other because of the small poloidal island width ( $\sim 10 \mathrm{~cm}$ on average) and the relatively-large connection lengths. The situation in the stochastic layer of LHD is even more complex, as shown in figure 10. The low-order island chains forming the stochastic layer have lower poloidal mode numbers (see figure 3 ). In addition, LHD has a much larger minor radius than W7-AS. The poloidal widths of the island chains are far beyond the cross-field transport length scale. Thus, friction between two 
neighbouring island fans within one (open) island chain is not expected for LHD. On the other hand, the large shear in LHD compresses the island chains of different poloidal mode numbers within a layer of $\sim 10 \mathrm{~cm}$ thickness. The islands belonging to different island chains are poloidally shifted with respect to each other. This phase-shift of the islands brings counter-flowing streams of plasma along neighbouring island chains into contact with each other. Locally-sheared flows have been indeed detected in LHD [45], as shown in the lower picture in figure 10.

\subsection{Extended two-point model}

The two terms on the right-hand side of eq (2) accounting for the momentum loss in helical SOLs have only a representative meaning. Of course, momentum transport is affected by the geometric details of the magnetic islands. The island form varies strongly in the helical direction and the islands interact with discontinuous targets. Thus, a quantitative assessment of the momentum loss needs 3D numerical modelling. For a qualitative understanding of the role of the momentum loss in divertor transport, we introduce a parameter $f_{m}$ to represent the integrated effect of the last two terms in eq (2) as

$$
p_{u}=2 p_{d}\left(1+f_{m}\right)
$$

Replacing the density $n$ in eq. (1) by the averaged upstream and downstream density, $\left(n_{e s}+n_{e d}\right) / 2$, we have from eq (1)

$$
T_{u p}^{7 / 2}=T_{d}^{7 / 2}+\frac{7 q_{\| 1} L_{c}}{2 \kappa_{e}}-\frac{7 \chi\left(n_{e s}+n_{e d}\right)}{4 \kappa_{e} \Theta^{2}}\left(T_{u p}-T_{d}\right)
$$

where $\chi=\chi_{i}+\chi_{e}$ and $\kappa_{i}$ is neglected against $\kappa_{e}$. Eqs (3), (5) and (6) form an extended two-point model for stellarators and reduce to a non-detached tokamak one when $f_{m} \rightarrow 0$ and $\Theta \rightarrow \infty$. For detached tokamak divertors a momentum loss term similar to $f_{m}$ appears also in tokamak 2-point models (see .e.g. [46]). But there, it stands for plasma-neutral friction.

We assume $f_{m}=0$ and $\Theta=0.1$ for (non-detached) tokamaks, and $f_{m}=\alpha / T_{d}^{1 / 2}$ [27] and $\Theta=0.001$ for stellarators with $\alpha$ being a free parameter representing the strength of the momentum loss. Then, for a given $q_{l l}$, the quantities $n_{e d}, T_{u p}$ and $T_{d}$ can be determined by eqs (3), (5) and (6) using $n_{e s}$ as an independent variable. For $q_{\| \prime}=0.5 \mathrm{MW} /\left(4 \pi^{2} a R \Theta\right)$ and $\chi=3 \mathrm{~m}^{2} / \mathrm{s}$, the results are shown in figure 11. The first dashed curve shows the standard two-point model results without cross-field transport as a reference. The sharp change in curve slope at $\mathrm{n}_{\mathrm{es}}=1 \times 10^{19} \mathrm{~m}^{-3}$ indicates the transition to the high-recycling regime. The solid curves show the results from the extended two-point model including cross-field transport for $\alpha=0,2,5$ and 10 . The small difference between the $\alpha=0$ curve and the standard case arises from the slight drop of $\mathrm{T}_{\text {up }}$ due to the cross-field heat conduction included in the extended model. With increasing $\alpha$ the relationship between $n_{e d}$ and $n_{e s}$ becomes increasingly linear, and the sharp transition from low to high recycling predicted by the standard two-point model disappears.

Figure 12 compares the EMC3-EIRENE simulation results for W7-AS, W7-X and ASDEX-Upgrade. Impurity radiation is not taken into account and the simulated plasmas correspond to an attached state without appreciable neutral-ion momentum loss. The same cross-field transport coefficients $\left(\mathrm{D}=1 \mathrm{~m}^{2} / \mathrm{s}\right.$ and $\left.\chi_{\mathrm{e}, \mathrm{i}}=3 \mathrm{D}\right)$ are assumed for the three devices, and the power entering the SOL $\left(\mathrm{P}_{\mathrm{SOL}}\right)$ is linearly scaled with the area of the LCFS, assuming the values of 1, 3, and $10 \mathrm{MW}$ for W7-AS, AUG and W7-X, respectively. The two curves for $\mathrm{W} 7-\mathrm{X}$ correspond to two cases with and without the control coils that can be used to fine-tune 
the internal pitch of field lines and thereby control the $\perp$-to-II transport ratio in the islands. The resulting effects are clearly shown in figure 12. With increasing control-coil current (Icc) and ensuing field-line pitch, the up-/downstream density correlation in W7-X approaches that predicted for the AUG tokamak. The high configurational flexibility of W7-X allows the divertor plasma to be controlled in a broad parameter range, thus bridging the gap between W7-AS and tokamaks (see figure 12).

\section{Impurity transport}

Reducing impurity release on plasma facing components and preventing impurities from entering the confinement core are crucial tasks of a divertor. Physical sputtering by bombardment of energetic ions and neutrals is one of the most critical impurity production processes. Thus, lowering the energy of the projectiles is essential for reducing the physical sputtering yield. As an electron-ion pair carries an amount $\gamma T_{d}$ of energy (see eq (3) where the ionisation energy of neutrals for the attached plasmas we are discussing is ignored, becoming however important for detached plasmas.), increasing the particle flux $n_{d} c_{s d}$ will reduce the energy of the ions hitting the targets. A high- $n_{d}$, low- $T_{d}$ plasma in the divertor region also provides favourable conditions for reducing the energy of the recycling neutral atoms and the resulting CX neutrals returning to the targets. Regarding the impurity and neutral screening, helical SOLs differ from a tokamak SOL in the following respects. (1) In a tokamak SOL, dense and cold plasmas under high recycling conditions are poloidally located in the divertor region. Moving upstream, the SOL plasma becomes "thinner" for CX-neutrals. The wall and other components in the main chamber face an upstream plasma in contact with the core. The helical, multiple-island structure in stellarators spreads out the downstream plasma over almost the entire SOL periphery in both the poloidal and toroidal directions. In other words, a large part of the SOL periphery facing the wall lies "downstream" and protects the wall from direct exposure to the hot "upstream" plasma. (2) Higher upstream density is needed for helical SOLs to achieve low downstream temperature, because of the geometry-related momentum loss discussed above. Generally, helical divertors can be operated at higher upstream densities than the poloidal divertor in tokamaks, as indeed was shown by both the W7-AS island divertor and the LHD helical divertor. (3) Parallel plasma flows are well distributed in helical SOLs (see figures 9 and 10) and are expected to be able to "flush out" impurities of different origins, including those originating from the wall and targets

For a collisional SOL and a sufficiently-small impurity concentration, the impurity transport along field lines is governed by the following force balance:

$$
0=m_{I} \frac{\left(V_{i \|}-V_{z \|}\right)}{\tau_{s}}+\beta_{i} \frac{d T_{i}}{d s}+\alpha_{e} \frac{d T_{e}}{d s}+Z e E-\frac{1}{n_{z}} \frac{d p_{z}}{d s}
$$

Eq (7) is widely used in the tokamak community for discussing impurity transport behaviour in the poloidal divertor and the meaning of the individual terms can be found in the literature (see, e.g. [4]). Using the simplified 1D geometry shown in figure 7 and assuming an anomalous cross-field diffusive process for impurities, the impurity continuity equation reads as

$$
\frac{d}{d x}\left(\Theta n_{z} V_{z \|}-D_{z} \frac{d}{d x} n_{z}\right)=S_{z}
$$

where $S_{z}$ represents the source of the ionization state $z$. The trace-impurity model included in 
the EMC3 code is based on eq (7) and (8) which are nevertheless solved in a realistic 3D space [25]. We split the impurity pressure gradient in temperature $\left(T_{z}=T_{i}\right)$ and density gradient and neglect the ion temperature gradient contribution with respect to the ion thermal force. Neglecting the electron thermal force and the electrostatic force in eq (7) and then inserting it into eq (8), one obtains

$$
\frac{d}{d x}\left[\Theta\left(V_{i l l}+\frac{\tau_{s} \beta_{i}}{m_{I}} \frac{d T_{i}}{d s}\right) n_{z}-\left(D_{z}+\Theta^{2} \frac{\tau_{s} T_{i}}{m_{I}}\right) \frac{d n_{z}}{d x}\right]=S_{z} .
$$

$\mathrm{Eq}(9)$ is valid for both tokamak and stellarator SOLs. For $m_{I}>>m_{i}$ (mass of background ions), $\beta_{i} \rightarrow 2.6 Z^{2}$ and then $\tau_{s} \beta_{i} / m_{I}=1.84 \tau_{i} / m_{i}$ with $\tau_{i}$ being the ion collision time. Note the parallel ion heat conductivity $3.9 n_{i} T_{i} \tau_{i} / m_{i}=\kappa_{i} T_{i}^{5 / 2}$ and let $D_{z / l}=\tau_{s} T_{i} / m_{I}$. Eq (9) can be rewritten as

$$
\frac{d}{d x}\left[\Theta\left(V_{i \|}+\frac{0.47}{p_{i}} \kappa_{i} T_{i}^{5 / 2} \frac{d T_{i}}{d s}\right) n_{z}-\left(D_{z}+\Theta^{2} D_{z \|}\right) \frac{d n_{z}}{d x}\right]=S_{z}
$$

where $p_{i}=n_{i} T_{i}$. In the following we assume a much shorter free path length of the sputtered impurity atoms in comparison to the recycling neutrals of the working gas, i.e. $S_{z}=0$, and separate the SOL into two regions according to the characteristic neutral penetration length $\lambda_{\mathrm{x}}$. In the first region within the distance $\lambda_{\mathrm{x}}$ to the target we assume $V_{i / l}=V_{i t h}$ (ion thermal velocity) and that the friction force dominates over the ion thermal force. Beyond $\lambda_{\mathrm{x}}, V_{i l l}=0$ and the ion thermal force dominates. This zone separation is done merely for simplifying our following analysis and discussion, but does not influence the main results. Ignoring the variation of $n_{i}$ and $T_{i}$ within $\lambda_{x}$, one obtains from eq (10)

$$
\ln \frac{n_{z, t}}{n_{z, \lambda x}}=\frac{\Theta V_{i t h} \lambda_{x}}{D_{z}+\Theta^{2} D_{z \|}}=\frac{V_{i t h} \lambda_{x}}{D_{z \|}} \frac{\Theta}{\Theta_{o p t}^{2}+\Theta^{2}} \text { with } \Theta_{o p t}=\sqrt{D_{z} / D_{z \|}} \text {. }
$$

where $n_{z, t}$ and $n_{z, \lambda x}$ are the impurity densities at the target and over the distance $\lambda_{x}$. For $\Theta$ $>\Theta_{o p t}, \quad \ln \left(n_{z, t} / n_{z, \lambda x}\right) \rightarrow V_{i t h} \lambda_{x} / \Theta D_{z l}=V_{i t h} \lambda_{\| I} D_{z l l}$ with $\lambda_{I F} \lambda_{x} / \Theta$ being the parallel neutral penetration length usually used in the tokamak literature. This situation is well understood for tokamaks. Because neutrals are not bound to magnetic field lines, the perpendicular distance $\lambda_{\mathrm{x}}$ is a more appropriate measure parameter than $\lambda_{l l}$ for characterizing the neutrals since the latter depends on the field-line pitch. From this point of view, eq (10) should have more general validity. It is interesting to note that the perpendicular transport and the field-line pitch $\Theta$ enter eq (11) in such a way that the ratio $\Theta /\left(\Theta_{\text {opt }}{ }^{2}+\Theta^{2}\right)$ has a maximum at $\Theta=\Theta_{\text {opt }}$. The physical explanation is following. If $\Theta \rightarrow 0$, the "radial" component of a parallel motion is negligible compared with the perpendicular transport, while for $\Theta \rightarrow 1 \lambda_{I /}$ approaches $\lambda_{x}$ and the length along which the fiction force can act on the impurities is shortened. Both weaken the retention effect of the friction force and the optimal case lies somewhere in between. For a given divertor configuration with fixed $\Theta$, its effect on the divertor retention capability of impurities does not have great practical significance, but it becomes of real interest when $\Theta$ can be varied or when configurations are compared where $\Theta$ varies by orders of magnitude.

$D_{z l l}$ is sensitive to $T_{i}\left(\propto T_{i}^{5 / 2}\right)$ and $\mathrm{Z}\left(\propto Z^{2}\right)$. We assume that the impurity downstream density is dominated by low charge-state impurity ions having $Z=2$ on average. For $n_{i}=$ $5 \times 10^{19} \mathrm{~m}^{-3}$ and $D_{z}=1 \mathrm{~m}^{2} / \mathrm{s}, \Theta_{\text {opt }}$ lies in the range of $0.009-0.07$ when $T_{i}$ varies from 50 to $10 \mathrm{eV}$. Taking the flux expansion effect into account and ignoring the 2D shaping of the targets, one finds that the poloidal divertor in tokamaks approaches the upper boundary of the $\Theta_{\text {opt }}$ 
window. In contrast, the small $\Theta$ in island SOLs is far below the "optimal range". Thus, it is to be expected that the friction force in the tokamak divertor geometry is more effective than in stellarators, at least for "out-flushing" the target-released impurities.

Let us now move upward beyond the neutral gas population zone into the second region where $V_{i l l}=0$. Recall eq (1) for ion heat transport and assume that the parallel ion heat flux is given by $q_{i l l}$. Then eq (10) can be rewritten as

$$
\frac{d}{d x}\left[-0.47 \frac{\Theta q_{\mathrm{ill}}}{p_{i}} \frac{\zeta_{i}}{1+\zeta_{i}} n_{z}-\left(D_{z}+\Theta^{2} D_{z \text { Ill }}\right) \frac{d n_{z}}{d x}\right]=0 .
$$

where $\zeta_{i}$ is the $I V \perp$ transport ratio for ions defined by eq (4). Because the ion thermal force is directed upstream toward increasing temperatures, impurities tend to accumulate at the SOL-core interface. If one attempts to approximate the poloidal divertor by assuming $\zeta_{i} \rightarrow \infty$ and $D_{z}<<\Theta^{2} D_{z l l}$, one would get a catastrophic build-up of upstream impurity density in tokamaks, which is recognized to be a physically unrealistic result because of the strictly $1 \mathrm{D}$ constraint. The problem is overcome by including a loss term on the right side of eq (12) to account for the radial transport of impurities into the SOL periphery, where it is assumed that impurities get lost without collisions. It is interesting to see that an equivalent perpendicular transport term appears naturally in eq (12) for helical SOLs. Now, we turn our attention from the diffusive term to the convective one because the latter is the "driver" of impurity leakage.

As the impurity flow velocity driven by the ion thermal force is associated with the classical ion heat flux [47], reducing the parallel ion heat flux is essential for reducing the impurity inward flow. This can be realized by e.g. decreasing the $q_{i l} / p_{i}$ ratio as shown in eq (12). Unfortunately, for a given divertor configuration, $q_{i l l}$ is linked to the heating power and the SOL parameter $p_{i}$ is limited usually by onset of thermal instabilities like Marfes [48, 49]. Eq (1) shows another possibility to reduce the classical heat flux, i.e. by means of cross-field transport. As shown in figure 8 , benefitting from the small value of $\Theta$, plasma conditions can be established in helical SOLs where the cross-field heat conduction dominates over the parallel one, i.e. $\zeta_{i}<1$. As $\zeta_{i} \propto T_{i}^{5 / 2} / n$, a further increase in $n$ and a resulting decrease in $T_{i}$ lead to a rapid decrease of $\zeta_{i}$ and the thermal-force induced impurity leakage flux (see eq (12)).

Impurity transport in the complex 3D SOLs of W7-AS, W7-X and LHD has been studied using the EMC3/EIRENE code. The simulation results have shown the existence of a friction-dominated impurity transport regime at high SOL collisionalities for all these devices [27,50-52]. Figure 13 shows how impurity density profiles throughout the stochastic layer in the LHD helical divertor change from peaked to hollow with increasing plasma separatrix density. The peaked profile at $\mathrm{n}_{\mathrm{es}}=2 \times 10^{19} \mathrm{~m}^{-3}$ reflects the governing role of the ion thermal force at low SOL collisionalities which draws the carbon impurities inward. The sudden profile flattening at higher densities in the inner SOL region is the consequence of a strong suppression of the ion thermal force. Once the thermal force is suppressed, a clear retention effect by friction emerges in the outer SOL region where most ionization of the recycling neutrals take place, as has been indeed observed experimentally [53].

Another example is shown for the standard island divertor of W7-X. Using the calculated background plasmas by the Icc $=25 \mathrm{kA}$ curve in figure 12 , test carbon and iron impurities are sampled on the targets (following the deposition distributions of the background ions) and wall (approximated by 20 points quasi-uniformly distributed over one 
half field period), respectively. Carbon atoms are started mono-energetically with $\mathrm{E}_{0}=0.1$ and $10 \mathrm{eV}$, which covers the energy range for chemical and physical sputtering processes. $\mathrm{Fe}$-atoms are initiated with $\mathrm{E}_{0}=5 \mathrm{eV}$. Figure 14 shows the dependence of the impurity density at the inner separatrix on $n_{\mathrm{es}}$ and $\mathrm{E}_{0}$. The sharp change in the curve-slope at $\mathrm{n}_{\mathrm{es}} \sim 2 \times 10^{19} \mathrm{~m}^{-3}$ indicates the transition from thermal-force to fiction dominated impurity transport in the SOL. A lower $n_{\mathrm{es}}$-boundary is set at $1 \times 10^{19} \mathrm{~m}^{-3}$ to exclude the low SOL collisionality cases where the ratio between the connection length and the ion/electron mean free path length is less than 10. As $\mathrm{n}_{\mathrm{es}}$ is increased to $2 \times 10^{19} \mathrm{~m}^{-3}$, this ratio increases sharply to 100 .

\section{Summary}

The divertor configurations currently explored in stellarators extend the parameter range far beyond that of the "traditional" poloidal divertor in tokamaks. In tokamaks, the nested magnetic flux surfaces are opened by introducing external poloidal fields of comparable strength to that generated by the plasma current. In low-shear stellarators, separatrix-bounded configurations are formed by natural magnetic islands created by much smaller radial perturbation fields inherently existing in the field spectrum of the 3D-shaped coils. The divertor potential of the magnetic islands arises from the internal field-line pitch associated with the shear. The helical divertor configuration in LHD is a natural product of the two helical coils. A pre-X-point stochastic layer of several-cm thickness forms the main part of the SOL which is expected to largely determine the divertor performance of the open HD. In the stochastic layer connection length ranges from $\mathrm{m}$ to $\mathrm{km}$. Generally, the divertor-relevant field-line pitch $\Theta$ (perpendicular displacement of a field line to targets per field-line length) in stellarators (for the LHD HD at least in the stochastic layer investigated) is much smaller than in tokamaks.

An extended two-point model is presented, taking both the parallel and cross-field transport into account, where $\Theta$ appears as a control parameter for the $\perp$-to-II transport ratio in the SOL. The model addresses the governing transport terms in both tokamaks and stellarators and clearly shows how their relative weights change when $\Theta$ varies from the tokamak to the stellarator level. In the most interesting SOL plasma parameter range, plasma transport in the poloidal divertor is governed by parallel transport processes. Decreasing $\Theta$ beyond the tokamak range, contributions of cross-field transport gradually increase and interesting effects emerge. Regarding impurity retention, the model shows that there exists an optimal field-line pitch $\Theta_{o p t}$ at which the friction force reaches its optimal effect in flushing the intrinsic impurities. $\Theta_{o p t}$ is determined by the $\perp$-to-II transport ratio of impurities, which is a function of the downstream plasma parameters. With $\Theta$ drops further down to the stellarator level, cross-field heat conduction can dominate over the parallel conduction for the ion energy transport throughout the islands. As the impurity flow velocity driven by the ion thermal force is associated with the classical conductive heat flux, having dominant cross-field heat conduction significantly reduces the thermal-force-driven inward flow of impurities. 3D simulations using the EMC3-EIRENE code based on a trace impurity model have predicted the existence of a friction-dominated impurity transport regime at high SOL collisionalities for all the devices that were investigated, W7-AS, W7-X and LHD. This has also been observed experimentally at high density in W7-AS and LHD.

Helical SOLs are fully three-dimensional and plasma transport is strongly modulated by the helical structure of the island chains. As a consequence, multiple parallel transport channels exist with varying shape and location, resulting in rather complex transport patterns, in particular for the parallel plasma flows. Counter-flows reside on different parts of the 
helical SOL, and cross-field transport can transfer momentum from one channel to another, causing momentum loss of counter-streaming ions. These geometry-induced effects make the essential transport features of helical SOLs deviate from the standard transport picture realized in tokamaks. For example, the high-recycling regime found in the tokamak poloidal divertor is not observed in W7-AS and LHD, nor is it expected from modelling. From another point of view, stellarators can be operated at higher upstream densities than tokamaks to reach comparably high density, low temperature plasma conditions at downstream.

Another important issue that is not addressed in the paper is the feasibility of thermal power removal via radiation under detachment. In tokamaks, it has been shown that the X-point geometry favours impurity radiation and a strong radiation belt around the X-point, a so-called MARFE, is usually observed in detached plasmas. The island chains in helical SOLs provide a multi-null divertor configuration where the number, location and geometry of the $\mathrm{X}$-points can be adjusted externally, thus having the potential of more flexible control of the radiation location for optimum power removal. Indeed, both W7-AS and LHD have demonstrated success in controlling and stabilizing the radiation layer outside the confinement region by externally manipulating the divertor geometry $[54,55]$. Numerically it is shown that the plasma-neutral interaction is not the main reason for the rollover of the recycling flux and downstream density in helical devices. The major features of the partially-detached plasma in W7-AS could be well explained by the EMC3-EIRENE code [26]. First simulation results for stable detachment in LHD have also shown similar tendency in carbon radiation pattern as observed experimentally [55]. The code typically shows asymmetric radiation patterns strongly correlated with the geometric details of the low-order magnetic islands. The relevant physics, however, has not been fully understood yet.

\section{References}

[1] Spitzer L 1951 US Atomic Energy Commission Report NYO-993 (PM-S-1)

[2] Spitzer L 1958 Phys. Fluids 1253

[3] Pitcher C S and Stangeby P C 1997 Plasma Phys. Control. Fusion 39779

[4] Stangeby P C 2000 The Plasma Boundary of Magnetic Fusion Devices, Plasma Phys. Ser., Institute of Physics Publishing

[5] Parker R R 1993 Plasma Phys. Control. Fusion B 4323

[6] Rebut P H et al 1993 Fus. Eng. Design 227

[7] Janeschitz G et al 1995 Plasma Phys. Control. Fusion 37 A19

[8] Kisslinger J et al 1995 Proc. 22nd EPS Conf. on Control. Fusion Plasma Phys. (Bournemouth, UK ) vol 19C

(Geneva: European Physical Society) (part III), p 149

[9] Grigull P et al 2001 Plasma Phys. Control. Fusion 43 A175

[10] Renner H et al 2000 Nucl. Fusion 401083

[11] Komori A et al., 2005 Nucl. Fusion 45, 837

[12] Morisaki T et al.,2005 J. Nucl. Mater. 337-339, 154

[13] Uo K et al., 1961 Journal of the physical society of Japan, vol.16 1380

[14] Ohyabu N et al 1994 Nucl. Fusion 34387

[15] König R et al. 2002 Plasma Phys. Control. Fusion 442365

[16] Braams B 1987 A multi-fluid code for simulation of the edge plasma in tokamaks NET Report EUR-FU IXII-80-87-68

[17] Rognlien T D et al 1992 J. Ncul. Mater 196-198 347

[18] Simonini R et al 1992 J. Ncul. Mater 196-198 369 
[19] Schneider R et al. 2006 Contrib. Plasma. Phys. 463

[20] R. Zagorski et al.,2008 Nucl. Fusion 48024013

[21] A. Runov et al., 2001 Phys. Plasmas 8916.

[22] Feng Y et al 1999 J. Ncul. Mater 266-269 812

[23] Reiter D et al 2005 Fusion Science and Technology 47172

[24] Feng Y et al. 2004 Contrib. Plasma. Phys. 4457

[25] Feng Y et al. 2002 Plasma Phys. Control. Fusion 44611

[26] Feng Y et al. 2005 Nucl. Fusion 4589

[27] Feng Y et al 2006 Nucl. Fusion 46807

[28] Wagner F et al 2005 Phys. Plasmas 12072509

[29] Hirsch M et al 2008 Plasma Phys. Control. Fusion 50053001

[30] Sharma D et al 2005 Nucl. Fusion 45825

[31] Kobayashi M et al 2007 J. Ncul. Mater 363-365 294

[32] Harting D et al 2008 Contrib. Plasma Phys. 48 (1-3) 1

[33] Frerichs H et al 2010 Computer Phys. Communication 18161

[34] Frerichs H et al 2010 Nucl. Fusion 50034004

[35] Kobayashi M 2007 Nucl. Fusion 4761

[36] Zha X et al 2009 J. Ncul. Mater 390-391 398

[37] Schmitz O et al 2008 Nucl. Fusion 48024009

[38] Masuzaki M et al 2002 Nucl. Fusion 42750

[39] Shimomura Y et al 1983 Nucl. Fusion 23869

[40] Kobayashi M et al., 2007 J. Nucl. Mater. 363-365 294

[41] Feng Y et al., 2008 Nucl. Fusion 48024012.

[42] Mahdavi A M et al 1981 Phys. Rev. Letters 47, 1602

[43] Borras K 1991 Nucl. Fusion 311035

[44] Stangeby P C 1993 Nucl. Fusion 331695

[45] Ezumi N et al., 2009 J. Plasma Fusion Res. SERIES, Vol. 8429

[46] Kotov V and Reiter D 2009 Phys. Control. Fusion 51115002

[47] Krashenninikov S. I. et al., 1991 Nucl. Fusion 31, 1455

[48] Baker D R et al 1982 Nucl. Fusion 22807

[49] Lipschultz B et al 1984 Nucl. Fusion 24977

[50] Kobayashi M. et al., 2009 J. Nucl. Mater. 390-391 325

[51] Feng Y et al., 2009 Nucl. Fusion 49095002

[52] Kobayashi M et al., 2010 Fusion Science and Technology vol. 58220

[53] Chowdhuri M. B. et al., 2009 Physics of Plasmas 16062502

[54] Grigull P et al., 2003 J. Nucl. Mater. 311-316 1287

[55] Kobayashi M et al., 2010 Phys. Plasmas 17056111 


\section{Figure captions}

Fig.1: The standard island divertor of W7-AS based on the 5/9 island chain. W7-AS has five field periods. Ten identical divertor modules of finite toroidal length are positioned up/down-symmetrically around the elliptical planes $\left(\varphi=36^{\circ}\right)$. The islands change their form toroidally. W7-X follows the same concept.

Fig. 2: Schematics of single-null and double-null tokamak divertors and the intrinsic island divertor for W7-AS and W7-X as well as the helical divertor for LHD. In W7-AS the poloidal model number $\mathrm{m}$ can be changed from 8-10, with 9 being the standard case, in W7-X $\mathrm{m}$ can be chosen from 4 to 6 , with $m=5$ being the standard case. The stochastic layer of the helical divertor in LHD consists of multiple low-order island chains.

Fig.3: Radially-zoomed connection length contour of the LHD HD with an overlying Poincare plot at a toroidal location where the long axis of the elliptical cross-section lies horizontally. The dashed lines indicate the expected locations of the low-order resonances. $r_{\text {eff }}$ is determined by cylindrical approximation of the volumes enclosed by the radial surface (elliptical form) in the computational domain. The four divertor legs are excluded.

Fig. 4: Dependences of divertor temperatures and density on line-averaged plasma density from an ohmic density scan in ASDEX [39].

Fig. 5: Right: Peak $\mathrm{n}_{\mathrm{ed}}$ values from target-integrated Langmuir probes at different locations versus the separatrix density $\mathrm{n}_{\mathrm{es}} . \mathrm{H}^{\mathrm{o}} \rightarrow \mathrm{H}^{+}$injection, $\mathrm{P}_{\mathrm{NI}}^{\text {abs }}=1.4 \mathrm{MW}$. Grey arrows indicate the directions of increasing the line-averaged density by gas-puffing. Left: EMC3-EIRENE simulation results with experimentally relevant input parameters. Here, $\mathrm{n}_{\mathrm{ed}}$ represents an average downstream density over all peaks in particle deposition weighted by energy flux density on targets.

Fig. 6: Ion saturation currents increase linearly with plasma density in LHD. This linear-dependence can be well reproduced by the 3D code.

Fig. 7: Schematic sketches of the single-null poloidal divertor in tokamaks (left) and the island divertors in stellarators (right) where $\Delta y$ indicates the width of a parallel flow channel.

Fig. 8: Relative weights of II and $\perp$ conductive heat fluxes of ions and electrons in typical SOL parameter ranges for stellarators $(\Theta=0.001)$ and tokamaks $(\Theta=0.1)$. The dashed arrow indicates a typical path of SOL plasma with increasing $\mathrm{n}_{\mathrm{e}}$.

Fig. 9: Calculated flow pattern for an attached plasma in the island divertor of W7-AS.

Fig. 10: Top: Calculated plasma flow contour in the stochastic layer of LHD. $r_{\text {eff }}$ on the vertical axis is the effective radius of elliptical mesh surfaces extended from core. Bottom: Comparison with the results of movable Mach probes [45] along the path indicated in the upper picture. 
Fig. 11: $\mathrm{n}_{\mathrm{ed}}$ vs. $\mathrm{n}_{\mathrm{es}}$ resulting from the extended two-point model with different strengths of momentum loss compared with standard two-point model for tokamaks (dashed line).

Fig.12: Comparison of divertor transport behavior in W7-AS, W7-X and ASDEX-Upgrade calculated by EMC3- EIRENE. $\mathrm{n}_{\text {ed }}$ is the downstream density whereas $\mathrm{n}_{\mathrm{es}}$ represents the average density on the LCFS.

Fig.13: Radial profiles of total carbon density $n_{I}$ normalized by downstream density $n_{I d}$ in ergodic layer of LHD for different background plasma densities, obtained from 3D modelling. $r_{\text {eff }}$ is defined in figure 3.

Fig. 14: SOL impurity retention capability as a function of SOL density predicted for the W7-X island divertor for target-released carbon and wall-released Fe. $\mathrm{E}_{0}$ : initial energy. $\mathrm{n}_{\mathrm{Is}}$ is impurity density at the inner separartrix normalized to $1 \mathrm{~A}$ yield for $\mathrm{C}$ and $\mathrm{Fe}$. 


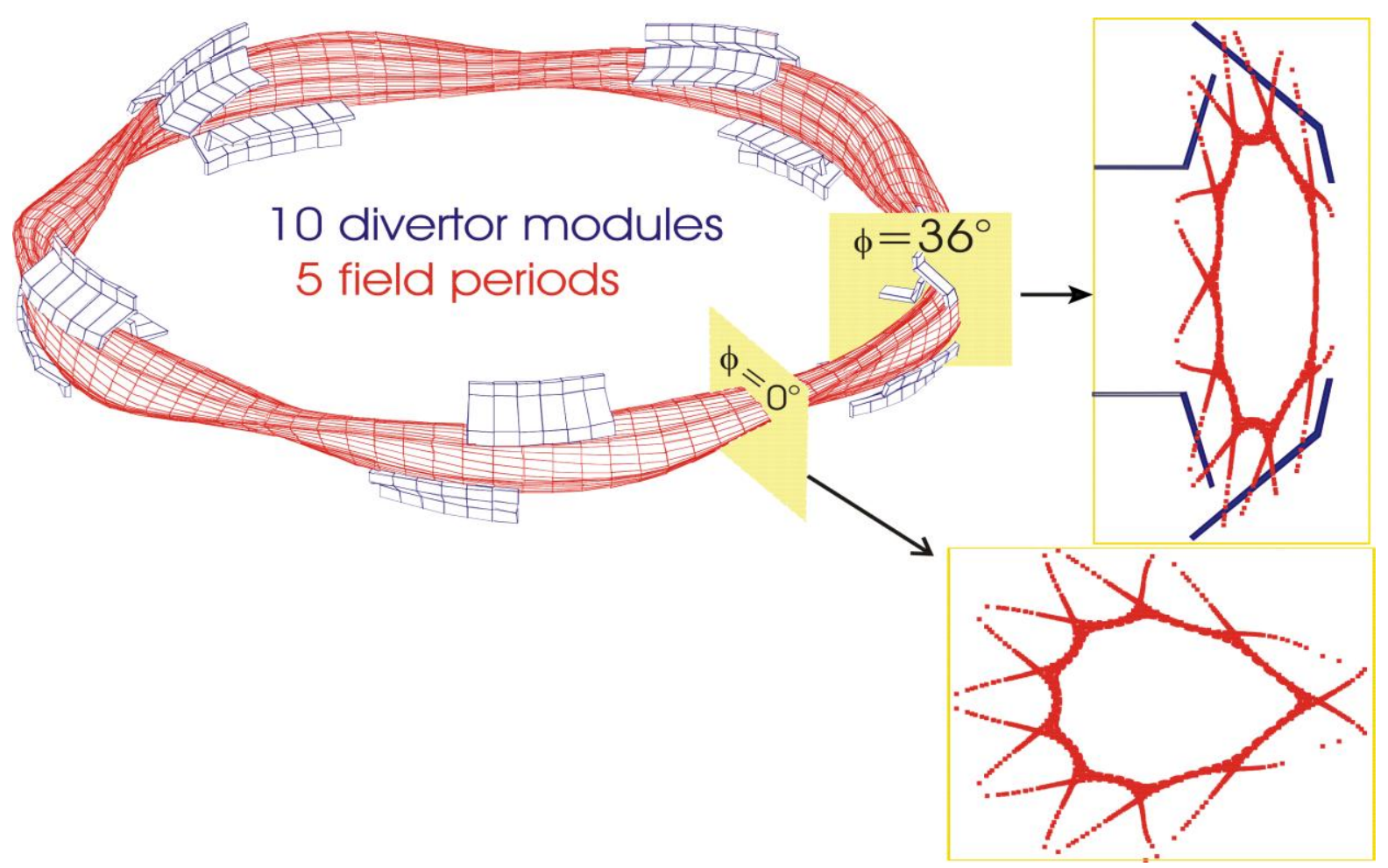

Figure 1 

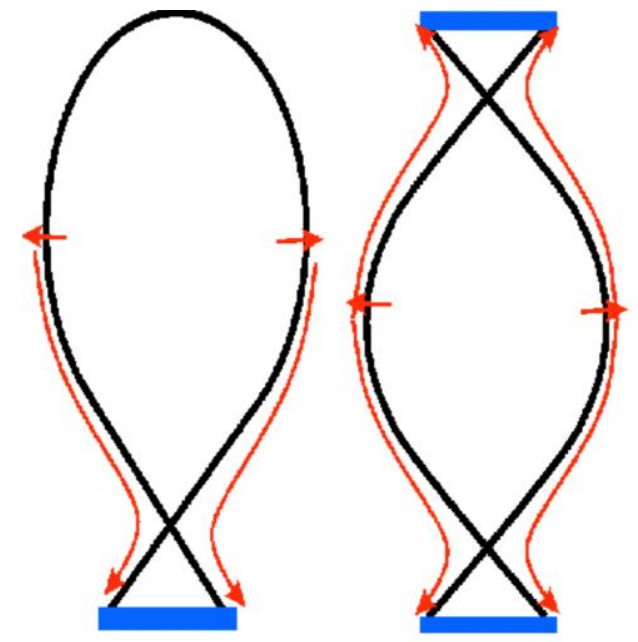

tokamaks

single null

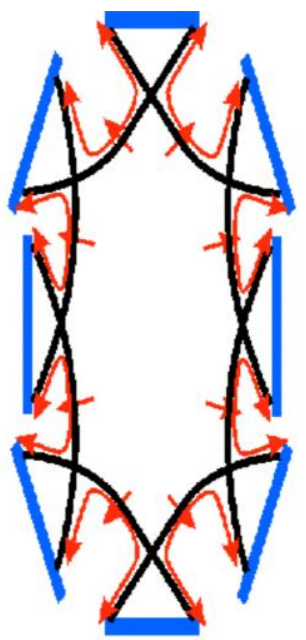

low-shear stellarator island divertor

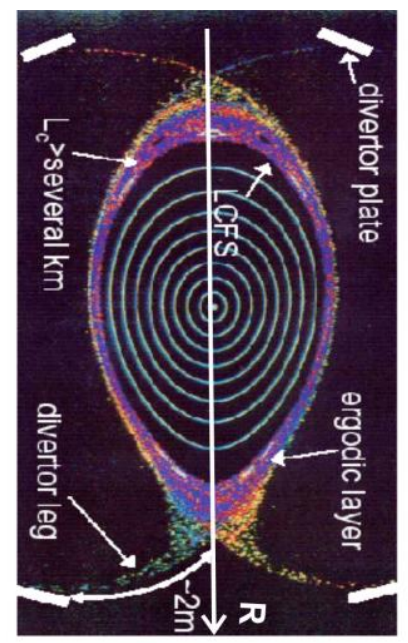

Heliotron/Torsatron helical divertor

Figure 2 


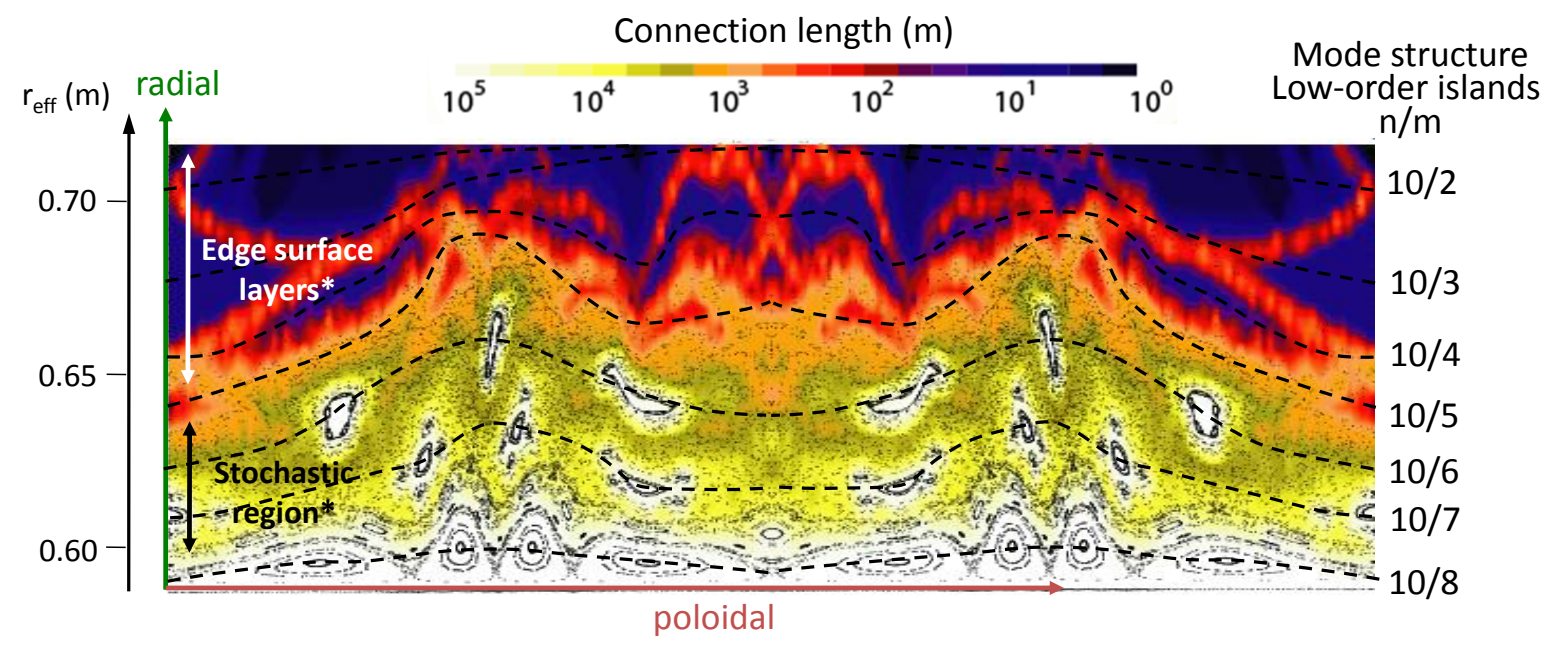

Figure 3 


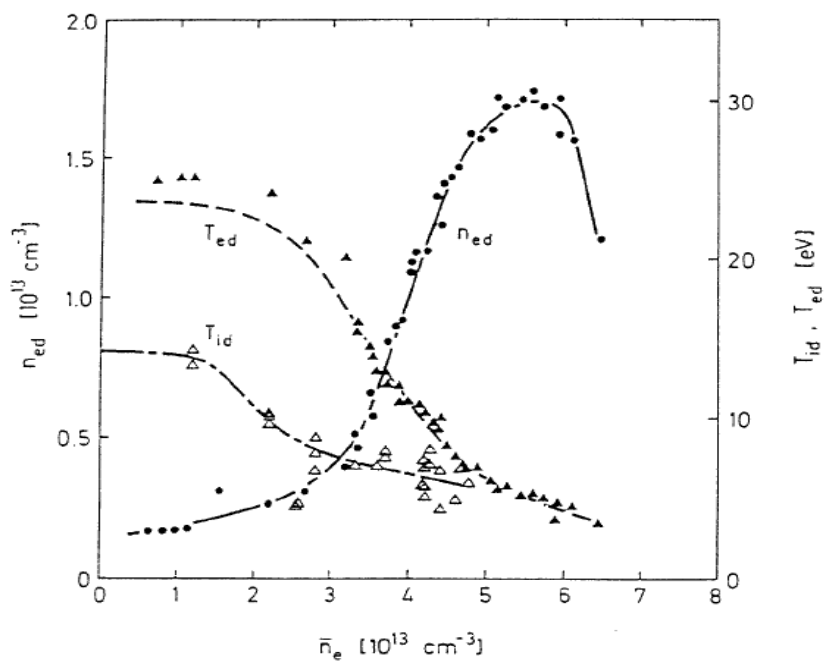

Figure 4 


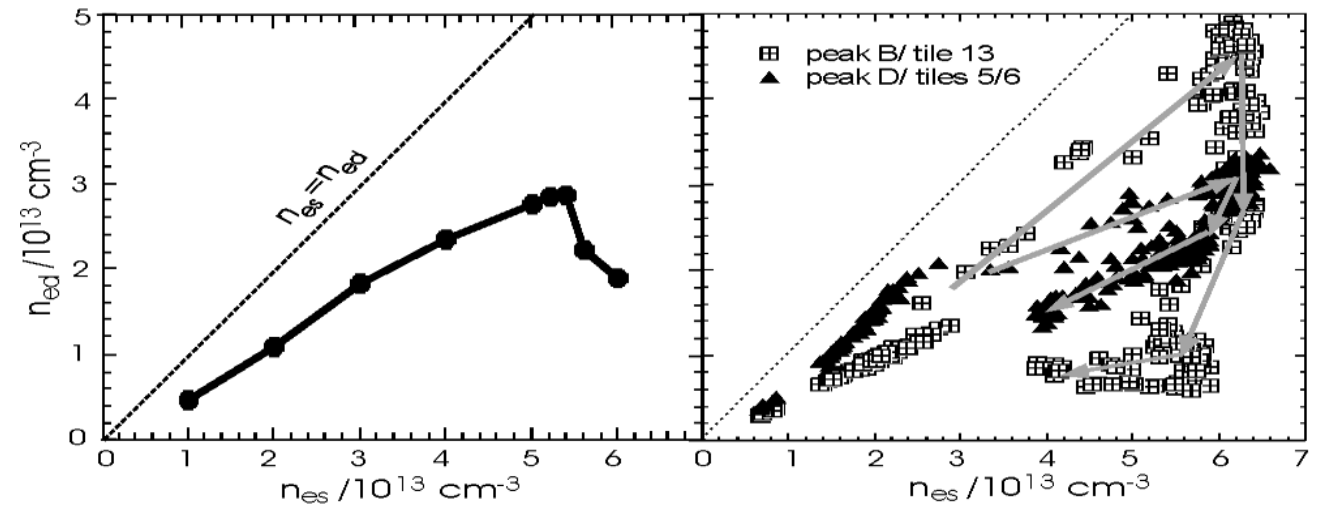

Figure 5 


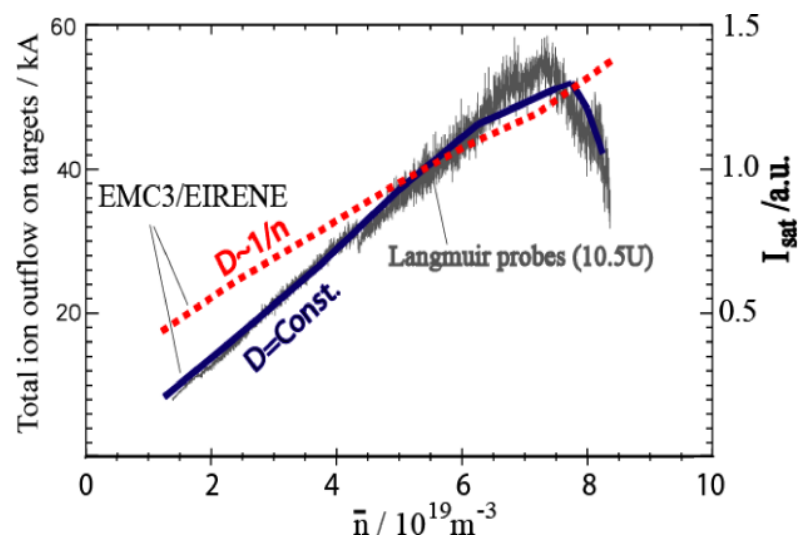

Figure 6 


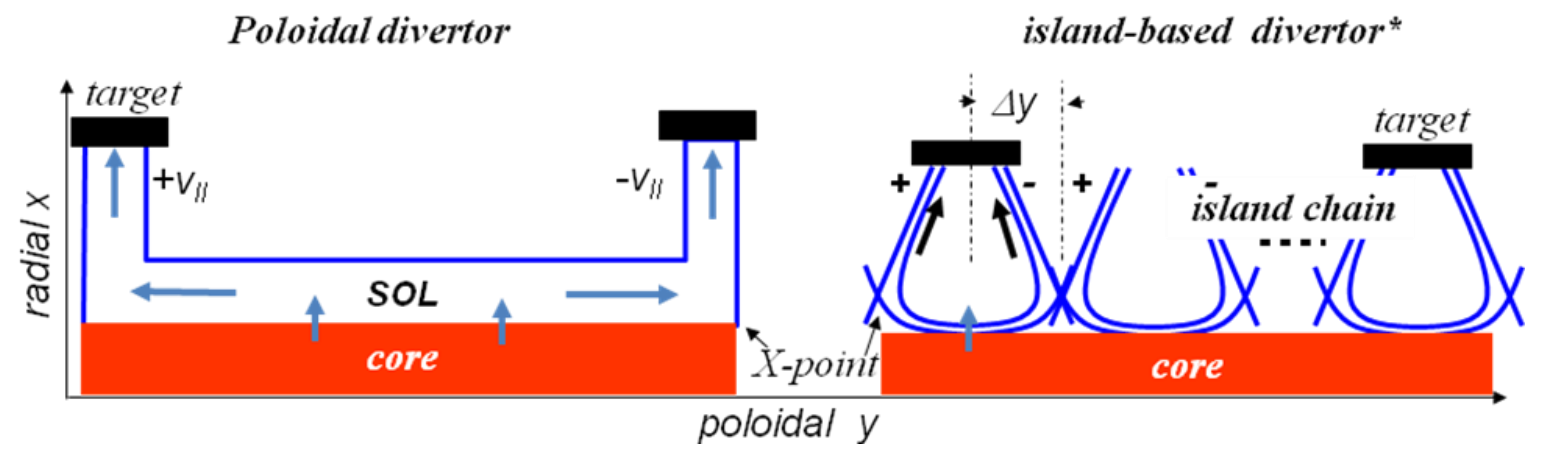

Figure 7 


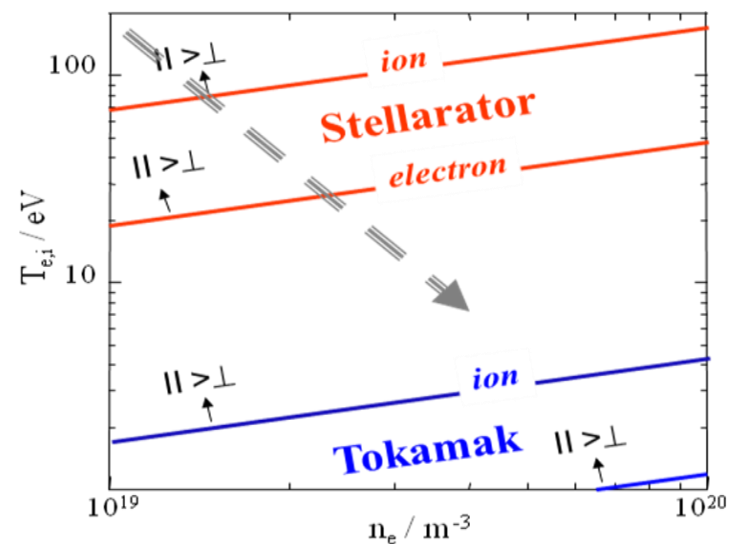

Figure 8 


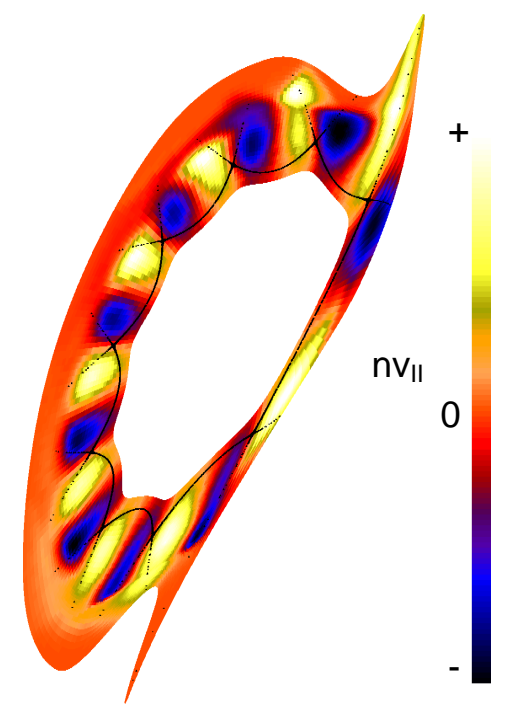

Figure 9 


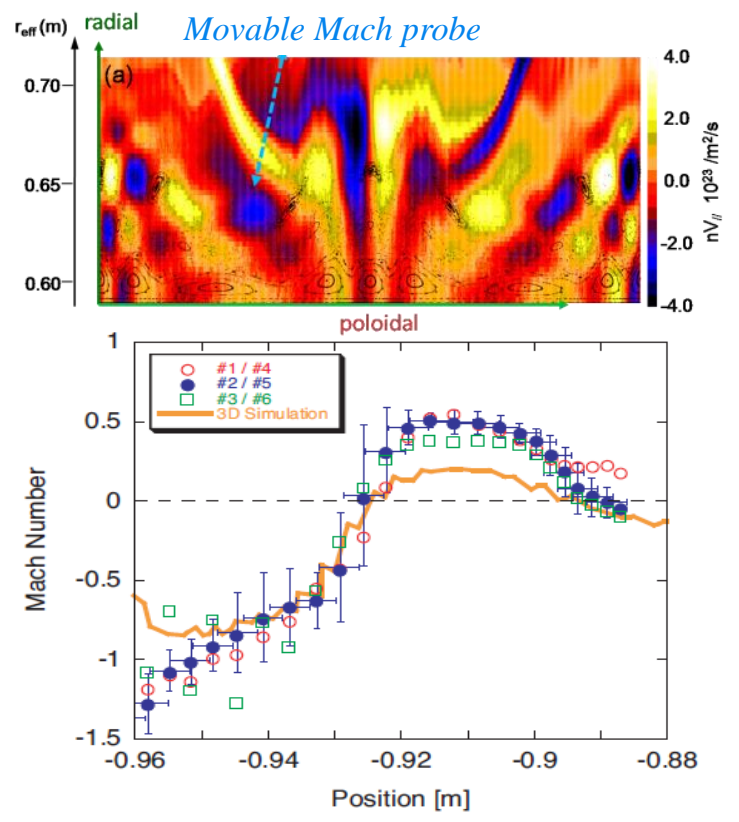

Figure 10 


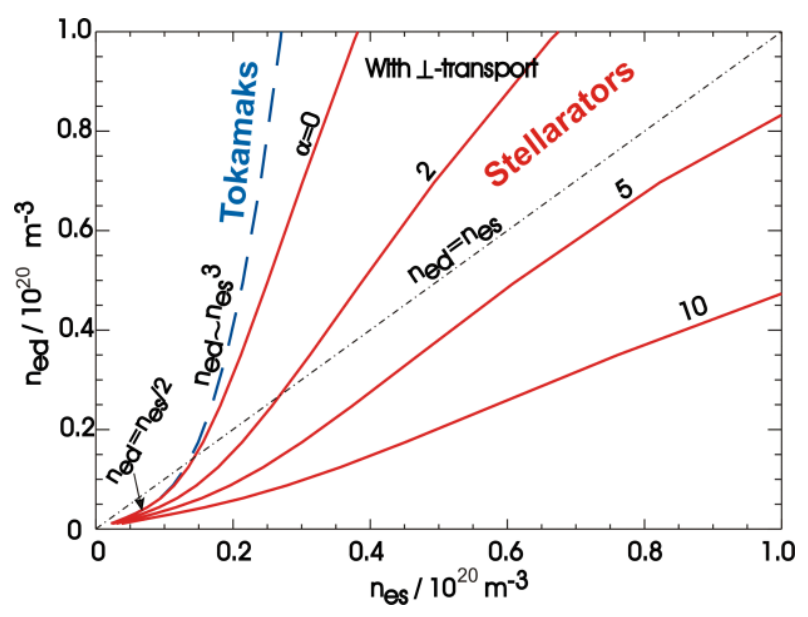

Figure 11 


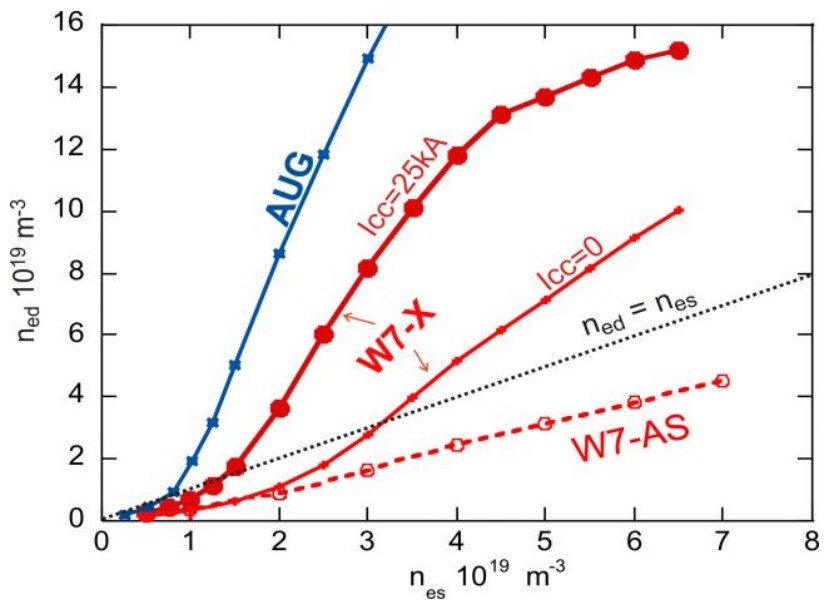

Figure 12 


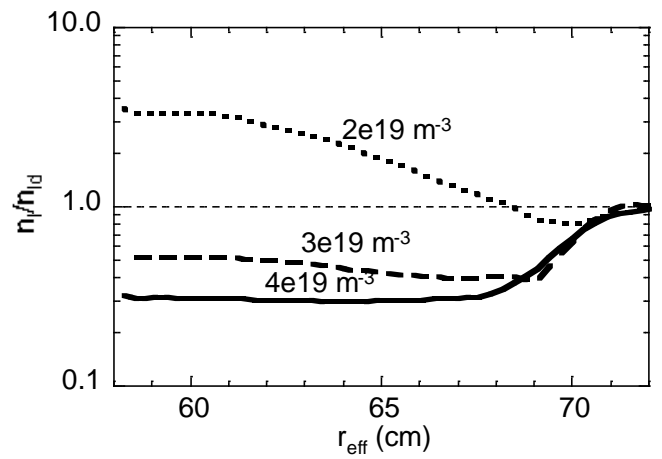

Figure 13 


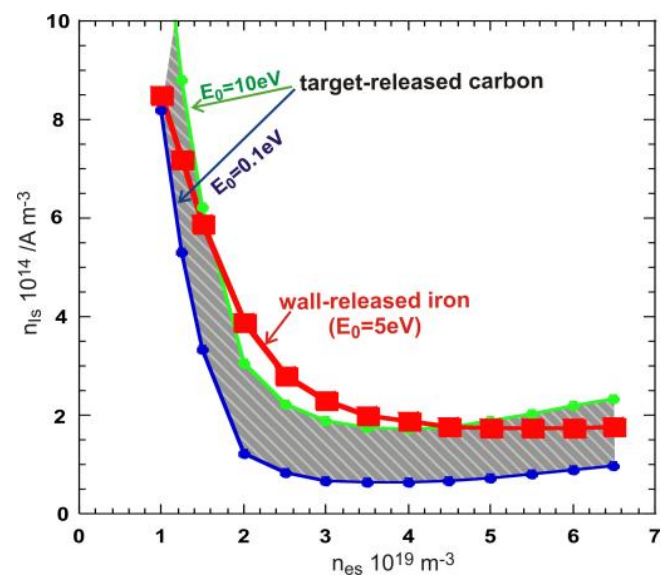

Figure 14 